\title{
C5aR is frequently expressed in metastatic renal cell carcinoma and plays a crucial role in cell invasion via the ERK and PI3 kinase pathways
}

\author{
YOSHIHIRO MAEDA $^{1 *}$, YOSHIAKI KAWANO ${ }^{1 *}$, YOSHIHIRO WADA ${ }^{1}$, JUNJI YATSUDA $^{1}$, \\ TAKANOBU MOTOSHIMA ${ }^{1}$, YOJI MURAKAMI ${ }^{1}$, KEN KIKUCHI $^{2}$, \\ TAKAHISA IMAMURA ${ }^{3}$ and MASATOSHI ETO ${ }^{1}$ \\ ${ }^{1}$ Department of Urology, Faculty of Life Sciences, Kumamoto University, Kumamoto 860-8556; \\ ${ }^{2}$ Medical Quality Management Center, Kumamoto University Hospital, Kumamoto 860-0811; \\ ${ }^{3}$ Department of Molecular Pathology, Faculty of Life Sciences, \\ Kumamoto University, Kumamoto 860-8556, Japan
}

Received November 20, 2014; Accepted December 19, 2014

DOI: $10.3892 /$ or.2015.3800

\begin{abstract}
The anaphylatoxin C5a is a chemoattractant for leukocyte migration via the $\mathrm{C} 5 \mathrm{a}$ receptor (C5aR). We recently reported that $\mathrm{C} 5 \mathrm{aR}$ was aberrantly expressed in a wide variety of human related cancers, while it also promotes cancer cell invasion by C5a stimulation. However, the biological significance of $\mathrm{C} 5 \mathrm{aR}$ expression in renal cell carcinoma (RCC) has not yet been clarified. In the present study, we aimed to elucidate the biological role of $\mathrm{C} 5 \mathrm{aR}$ in $\mathrm{RCC}$ progression. Clinical RCC specimens were analyzed for C5aR expression and its relationship with baseline demographic data and clinicopathological parameters was analyzed. Moreover, renal carcinoma Renca cells stably expressing C5aR were generated and used to assess the effects of C5a-C5aR axis activation on various cellular phenomena in culture. Immunohistochemistry revealed that $96.7 \%$ of the metastatic RCCs (mRCCs) showed C5aR expression, whereas only $50.5 \%$ of the non-metastatic RCCs expressed C5aR ( $\mathrm{P}<0.001)$. Although C5a stimulation did not significantly alter anoikis of C5aR-expressing Renca cells, C5a elicited cell morphological change and scattering of those cells accompanied with dynamic actin rearrangement, which was not observed in the Renca cells harboring the empty vector only. Moreover, C5a triggered ERK and PI3K-dependent invasion of the C5aR-expressing renal carcinoma cells. These results are consistent with the idea that the C5a-C5aR axis
\end{abstract}

Correspondence to: Dr Yoshiaki Kawano, Department of Urology, Faculty of Life Sciences, Kumamoto University, 1-1-1 Honjo, Kumamoto, Kumamoto 860-8556, Japan

E-mail: ykawano@kumamoto-u.ac.jp

"Contributed equally

Key words: renal cell carcinoma, $\mathrm{C} 5 \mathrm{a}$ receptor, signal transduction, neoplasm invasiveness plays a crucial role in renal carcinoma cell invasion, which may be one of the key steps for RCC metastasis. The present study provides proof-of-concept that the C5a-C5aR axis may be a useful therapeutic target for preventing RCC progression.

\section{Introduction}

Renal cell carcinoma ( $\mathrm{RCC}$ ) is the most common type of kidney cancer in adults. Surgical removal of the cancer, either by radical or partial nephrectomy, is a standard therapeutic option for organ-confined RCC. However, $30 \%$ of patients eventually manifest metastatic disease after surgery (1). Recent studies have clarified the key molecular events of oncogenesis for renal cell carcinoma such as aberrant hypoxia-inducible factor (HIF)- $\alpha$ activation by Von Hippel-Lindau (VHL) gene mutation/deletion and the resultant overexpression of vascular endothelial growth factor (VEGF) in clear cell carcinoma, which provided the rationale for the development of VEGF receptor (VEGFR) inhibitors such as sunitinib and sorafenib. Although contemporary emergence of such molecular targeting agents has contributed to the prolonged survival of metastatic RCC (mRCC) patients, attainment of complete response is extremely rare (2) and improvement in overall survival is still limited. Therefore, detailed mechanisms of how RCC cells spread and eventually metastasize must be clarified in order to develop better therapeutic options.

Cancer cell invasion and metastasis share many similarities with leukocyte trafficking, which is crucially regulated by soluble factors and their receptors (3). Anaphylatoxin C5a is an $\mathrm{N}$-terminal 74 amino acid fragment derived from the $\alpha$-chain of the complement fifth component (C5), which serves as a leukocyte chemoattractant and inflammatory mediator (4). C5a promotes leukocyte migration by interacting with the membrane-associated C5a receptor (C5aR; CD88). C5aR is one of the G protein-coupled receptors (GPCRs), and its association with the $\mathrm{C} 5 \mathrm{a}$ ligand provokes activation of intracellular signaling pathways such as the Raf/MEK/ERK and 
PI3K/Akt pathways $(5,6)$, which play pivotal roles in leukocyte migration $(7,8)$. Recently, we reported that C5aR is aberrantly expressed in a wide variety of human cancers presumably due to the consequence of malignant transformation while the C5a-C5aR axis promoted cancer cell invasion by eliciting matrix metalloprotease (MMP) secretion and cytoskeletal reorganization (9). In that study, we briefly mentioned that C5aR was also expressed in renal cell carcinoma specimens. However, the number of specimens in that study was limited, and the clinical significance and biological role of the C5aR expression in renal cell carcinoma remains unclear.

Here, we analyzed the relationship between the C5aR expression and the clinical parameters in renal cell carcinoma specimens. We observed that C5aR was expressed in a vast majority of the $\mathrm{mRCC}$ cases, whereas only half of the organ-confined RCCs expressed C5aR. Furthermore, we provided in vitro evidence that the C5a-C5aR axis provoked renal cancer cell invasion. Our results suggest that the C5a-C5aR axis-elicited renal cancer cell invasion may be one of the critical steps for establishing renal cancer cell metastasis.

\section{Materials and methods}

Cell line. Renca cells (ATCC: CRL-2947) were obtained in 2013 from the American Type Culture Collection. The cells were cultured in RPMI-1640 supplemented with $10 \%$ fetal calf serum (FCS), penicillin $(40 \mathrm{U} / \mathrm{ml})$ and streptomycin $(40 \mu \mathrm{g} / \mathrm{ml})$ and were maintained at $37^{\circ} \mathrm{C}$ in $5 \% \mathrm{CO}_{2}$.

Reagents. Recombinant C5a was purchased from Hycult Biotech (Plymouth, PA, USA). G418 was purchased from InvivoGen (San Diego, CA, USA). U0126 was purchased from Promega (Madison, WI, USA). PI-103 was purchased from Merck (Darmstadt, Germany). Anti-C5aR antibody was purchased from Santa Cruz Biotechnology (Dallas, TX, USA). Antibodies for phospho-ERK and total ERK, phospho-Akt and total Akt and HRP-conjugated secondary antibodies were purchased from Cell Signaling Technology (Danvers, MA, USA).

Tissue samples, immunohistochemistry and retrospective analysis. Renal cell carcinoma tissue samples were obtained by surgical resection or core needle biopsy from 127 patients in Kumamoto University Hospital, and the usage of those samples for the present study was approved by The Internal Review Board of Kumamoto University Hospital. Immunohistochemistry for C5aR in the RCC samples was performed according to a previously described protocol (9). The relationship between the C5aR expression and baseline demographic data/clinicopathological parameters was analyzed by Fisher's exact test.

Immunoblotting. Immunoblotting was performed as previously described (10). Briefly, cell lysates were analyzed by SDS-PAGE under reducing conditions and transferred to nitrocellulose membranes (Protran; GE Healthcare Life Sciences, Pittsburgh, PA, USA). After blocking with blocking buffer (1\% TBS-T buffer containing 5\% bovine serum albumin), the membranes were incubated with the primary antibodies indicated according to the manufacturer's instructions, followed by incubation with appropriate HRP-conjugated secondary antibodies. The bands were visualized by ECL Plus Western Blotting Detection System (GE Healthcare Life Sciences) according to the manufacturer's instructions.

Establishment of C5aR stably expressing Renca cells and in vivo study. pCMV-C5aR that encodes full-length murine C5aR was purchased from OriGene Technologies (Rockville, MD, USA). Renca cells were transfected with pCMV-C5aR using the ProFection Mammalian Transfection System (Promega). After $48 \mathrm{~h}$, the medium was replaced with a selection medium supplemented with G418 $(400 \mu \mathrm{g} / \mathrm{ml})$ then cultured for 2 weeks. G418-resistant cells were isolated by limiting dilution and propagated. The cells were subjected to flow cytometric analysis by FACSCan (BD Biosciences, San Jose, CA, USA) as previously described (9) to select cells expressing C5aR (designated as Renca/C5aR cells). Renca cells transfected with the empty plasmid pCMV and resistant to $400 \mu \mathrm{g} / \mathrm{ml} \mathrm{G} 418$ were isolated and then designated as Renca/empty cells. For the in vivo study, the Renca-derived cells were injected into the renal subcapsular space in mice according to Shvarts et al (11). This experiment was approved by the Kumamoto University Animal Experiment Committee.

Anoikis assay. Anoikis assay was performed based on the protocol reported by Berezovskaya et al (12) with minor modifications. Renca-derived cells were dissociated by Accutase (Millipore, Billerica, MA, USA), and then washed with serum-free medium and suspended with medium containing $0.5 \%$ FCS. The viable cells were counted using the trypan blue dye exclusion method to confirm that the initial viability of the cells after dissociation by Accutase was $>95 \%$. A suspension of $1 \times 10^{6}$ viable cells was treated with or without $10 \mathrm{nM}$ recombinant $\mathrm{C} 5 \mathrm{a}$ ( $\mathrm{rC5}$ a) then plated in $2.0 \mu \mathrm{l}$ of serum-free medium in 6-well ultra-low-attachment polystyrene plates (Corning, Tewksbury, MA, USA) and incubated at $37^{\circ} \mathrm{C}$ in $5 \% \mathrm{CO}_{2}$ overnight. The numbers of the total and viable cells after incubation were counted as described above, and then the percentage of the viable cells was calculated.

Immunofluorescence analysis. Cells $\left(5 \times 10^{4}\right)$ were seeded on glass coverslips and incubated for $24 \mathrm{~h}$. After serum starvation overnight, the cells were stimulated with $10 \mathrm{nM} \mathrm{rC5a}$ for the stated time periods. The cells were then fixed in $4 \%$ paraformaldehyde, permeabilized in $0.2 \%$ Triton X-100 for $5 \mathrm{~min}$, and were incubated with $5 \mathrm{U} / \mathrm{ml}$ Alexa 488-phalloidin (Invitrogen Life Technologies, Carlsbad, CA, USA) for $40 \mathrm{~min}$, followed by washing with PBS. Nuclei were counterstained with $1.0 \mathrm{mM}$ TO-PRO ${ }^{\circledR}-3$ (Invitrogen Life Technologies) for $15 \mathrm{~min}$. Images were obtained and processed by FluoView 300 laser scanning confocal microscope (Olympus, Melville, NY, USA).

Invasion assay in vitro. BioCoat Matrigel invasion chambers were utilized (24-well plates, $8-\mu$ m pores; BD Biosciences) as previously described (9). Renca-derived cells $\left(5 \times 10^{4}\right)$ were suspended in serum-free RPMI-1640, which were then seeded into the upper chamber. RPMI-1640 supplemented with either $10 \mathrm{nM}$ rC5a or carrier solution (PBS) was placed in the lower chamber. PI-103, U0126 or carrier control (DMSO) was added to the medium in both the upper and lower well at the indi- 
Table I. Patients and tumor characteristics.

\begin{tabular}{|c|c|c|c|}
\hline & $\begin{array}{l}\text { C5aR(-) } \\
(n=49)\end{array}$ & $\begin{array}{c}\text { C5aR(+) } \\
(\mathrm{n}=78)\end{array}$ & P-value \\
\hline Age (years) & 61.8 & 61.490 & 0.8962 \\
\hline \multicolumn{4}{|l|}{ Gender, n (\%) } \\
\hline Male & $36(73.5)$ & $50(64.1)$ & 0.3313 \\
\hline Female & $13(26.5)$ & $28(35.9)$ & \\
\hline \multicolumn{4}{|l|}{$\begin{array}{l}\text { Histological } \\
\text { subtypes, n (\%) }\end{array}$} \\
\hline Clear cell & $48(100)$ & $72(92.3)$ & 0.0812 \\
\hline Chromophobe & $0(0)$ & $3(3.8)$ & 0.2835 \\
\hline Papillary & $0(0)$ & $3(3.8)$ & 0.2835 \\
\hline \multicolumn{4}{|c|}{ Fuhrman grade, n (\%) } \\
\hline G1 & $23(46.9)$ & $17(21.8)$ & 0.0056 \\
\hline $\mathrm{G} 2$ & $24(49.0)$ & $50(69.2)$ & 0.0259 \\
\hline G3 & $1(2.0)$ & $5(6.4)$ & 0.4044 \\
\hline Unknown & $1(2.0)$ & $2(2.6)$ & NA \\
\hline \multicolumn{4}{|l|}{ Stage $^{\mathrm{a}}, \mathrm{n}(\%)$} \\
\hline $\mathrm{T} 1$ & $46(93.9)$ & $55(70.5)$ & 0.0056 \\
\hline $\mathrm{T} 2$ & $2(4.1)$ & $10(12.8)$ & 0.1270 \\
\hline $\mathrm{T} 3$ & $1(2.0)$ & $11(14.1)$ & 0.0283 \\
\hline $\mathrm{T} 4$ & $0(0)$ & $2(2.6)$ & NA \\
\hline No & $48(98.0)$ & $68(87.2)$ & 0.0497 \\
\hline N1 & $0(0)$ & $5(6.4)$ & 0.1555 \\
\hline $\mathrm{N} 2$ & $1(2)$ & $5(6.4)$ & 0.4044 \\
\hline M0 & $48(98.0)$ & $49(62.8)$ & $<0.0001$ \\
\hline M1 & $1(2)$ & $29(37.2)$ & \\
\hline \multicolumn{4}{|l|}{$\begin{array}{l}\text { Microscopic } \\
\text { invasion }^{\mathrm{b}}, \mathrm{n}(\%)\end{array}$} \\
\hline Vascular & $3(6.3)$ & $20(29.0)$ & 0.0038 \\
\hline Lymphatic & $0(0)$ & $3(4.7)$ & 0.2587 \\
\hline
\end{tabular}

Significant P-values are indicated in bold style. NA, not available. ${ }^{a} \mathrm{~T}$ staging was based upon the pathological assessment of surgical specimen except for a single case, for which histology (clear cell subtype) was diagnosed by needle biopsy and staging (T4) were radiographically assessed. $\mathrm{N}$ and $\mathrm{M}$ staging are based upon radiographical assessment. ${ }^{b}$ Due to diagnostic limitation, not all cases were available for assessment (a: 48 cases, b: 69 cases and c: 64 cases). C5aR, C5a receptor.

cated concentrations when appropriate. The numbers of cells that migrated through the membrane were counted in 4 microscopic fields (x20 magnification) per membrane. The average was calculated from triplicate samples, and statistical analyses were performed by two-tailed t-tests.

\section{Results}

Frequent expression of the C5a receptor in metastatic renal cell carcinoma. First, we analyzed C5aR expression in 127 primary RCC specimens using surgically removed or needle biopsy samples. Overall, the C5aR expression was observed in 78 out of 127 samples (61.4\%). This sample cohort consisted of 97 RCCs without metastasis and $30 \mathrm{mRCC}$ (Fig. 1). As shown in Table I, there was no significant difference between the C5aR-positive and -negative group in regards to age, gender and histological subtype. Regarding Fuhrman grade, although the grade 3 population was quite low $(n=6)$ in this sample cohort, C5aR-positive tumors tended to exhibit a higher grade than the C5aR-negative tumors (G1: 21.8 vs. $46.9 \%$, G2: 69.2 vs. 49.0\%). Regarding $\mathrm{T}$ staging, the C5aR-positive group contained significantly less $\mathrm{T} 1$ tumors $(\mathrm{P}=0.0056)$ and more T3 tumors $(\mathrm{P}=0.0283)$ than the $\mathrm{C} 5 \mathrm{aR}$-negative group. As for $\mathrm{N}$ staging, more $\mathrm{C} 5 \mathrm{aR}$-negative tumors were without lymph node metastasis (N0) than the C5aR-positive tumors (98.0 vs. $87.2 \%)$ with marginal significance $(\mathrm{P}=0.0497)$. Distant metastasis was more frequently observed in the C5aR-positive group than in the C5aR-negative group (37.2 vs. $2 \%$ ). Of note, $96.7 \%$ (29/30) of the patients with metastatic disease showed C5aR expression at their primary sites. With regards to microscopic invasion, more C5aR-positive tumors manifested microvascular invasion than C5aR-negative tumors (29.0 vs. 6.3\%). These results suggest that $\mathrm{C} 5 \mathrm{aR}$ expression contributes to local invasion and distant metastasis of renal cell carcinoma.

C5a-C5aR axis is not involved in Renca cell proliferation and anoixis. To study the biological role of the C5a-C5aR axis in renal cell carcinoma, we employed Renca cells since these cells are the established model for studying renal cell carcinoma metastasis $(11,13)$. Interestingly, both western blotting and flow cytometric analysis showed that C5aR was not expressed in Renca cells (data not shown). Such an observation in cancer cell lines, which does not seemingly reflect the characteristics in clinical specimen, was described and discussed in our previous study, suggesting that C5aR expression was lost during the process of cell line establishment from primary culture of cancer cells in order to prioritize the expression of other essential proteins for clonal development in the context of 2-dimensional culture (9). To establish renal cell carcinoma cells expressing C5aR, C5aR cDNA was stably introduced into Renca cells, and clones expressing C5aR were isolated and propagated. Cells expressing $\mathrm{C} 5 \mathrm{aR}$ on their cell surface, confirmed by flow cytometric analysis (Fig. 2A), were selected and designated as Renca/C5aR cells. Cells stably transfected with the empty plasmid were designated as Renca/empty cells. The original purpose of establishing Renca/C5aR cells was to study if C5aR can enhance the metastatic behavior of renal carcinoma cells in a syngeneic orthotopic murine model using $\mathrm{BALB} / \mathrm{c}$ mice since Renca cells are known to establish multiple lung metastasis in this model (11). However, the Renca/C5aR cells, which were stimulated by recombinant C5a and then injected into the renal subcapsular space, did not exhibit any increase in either the number or the size of lung metastatic nests compared to the Renca/empty cells (data not shown). This may be due to the difficulty of attaining sustained C5aR activation by recombinant $\mathrm{C} 5 \mathrm{a}$ until establishing lung metastasis, considering the stability of $\mathrm{C} 5 \mathrm{a}$ and the period required for tumor cell implantation into the lungs after subcapsular injection. Because of such technical difficulty of the in vivo study to analyze the biological role of C5aR expression in renal cell carcinoma metastasis, we analyzed the effect of C5aR on crucial steps of cancer metastasis in vitro instead. Anoikis is a subtype of apoptosis induced upon cell detachment from the extracellular matrix, which is an indispensable step for 

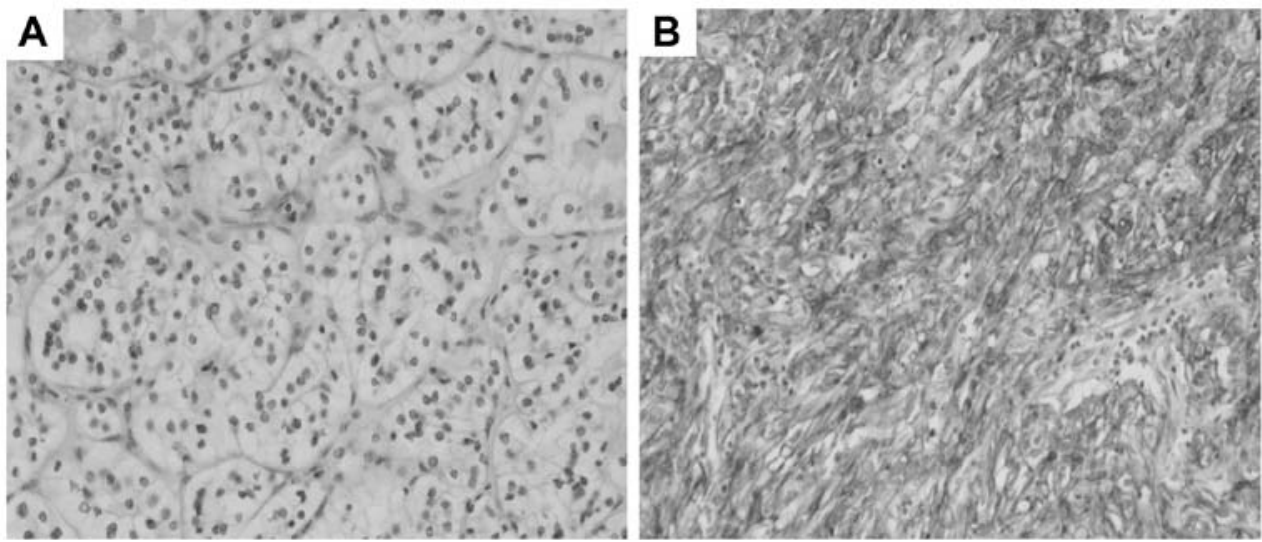

Figure 1. Immunohistochemistry of C5aR in the RCC specimens. FFPE samples of RCC were stained with the anti-C5aR antibody. Representative examples of C5aR-negative RCC without metastasis (A) and C5aR-expressing mRCC (B) are shown. Original, x200 magnification. C5aR, C5a receptor; RCC, renal cell carcinoma; mRCC, metastatic RCC; FFPE, formalin-fixed paraffin-embedded.

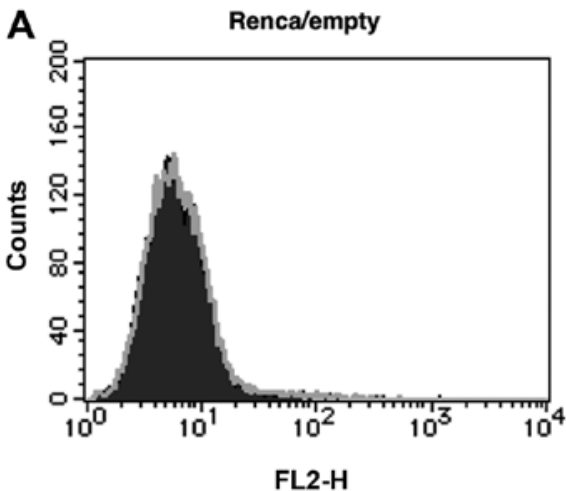

B

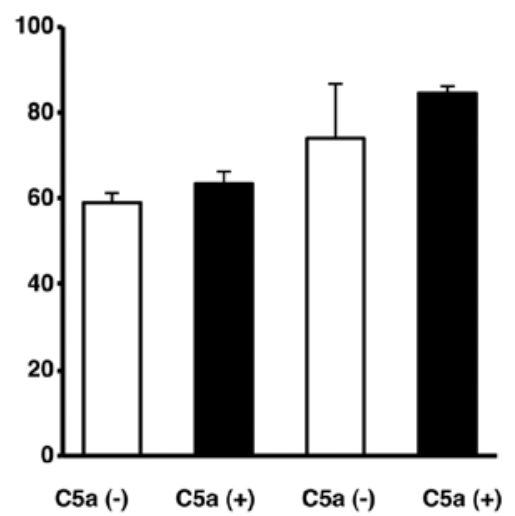

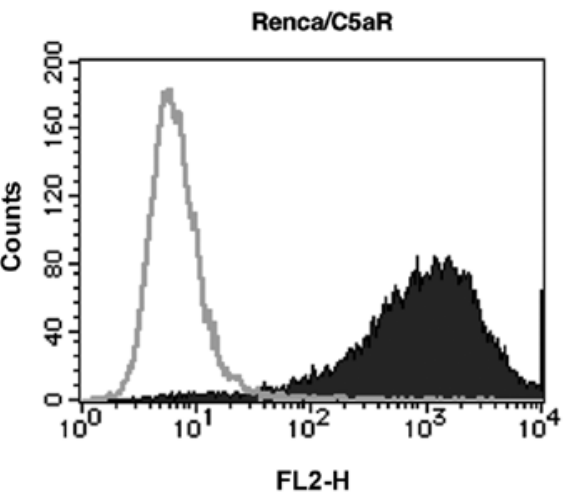

Figure 2. (A) Establishment of Renca cells stably expressing C5aR or the empty plasmid. C5aR-derived cells were examined for C5aR surface expression using PE-conjugated anti-mouse C5aR antibody (filled area). Isotype matched control antibody was used as a negative control (grey line). The abscissa represents the relative fluorescence intensity and the ordinate the relative cell number (counts). (B) C5a-C5aR axis is not involved in anoixis resistance by Renca cells. Renca-derived cells were treated with or without C5a and cultured in a floating condition. The bar graph represents the percentage of viable cells. C5aR, C5a receptor. metastasis (14). To analyze whether renal carcinoma cells are able to acquire resistance to anoikis by C5a-C5aR axis activation, Renca-derived cells were treated with or without C5a and then cultured in suspension on ultra low-attachment plates and a number of viable cells was assessed. Fig. 2B shows that the percentage of Renca/C5aR cell survival in suspension culture was slightly higher than that of the Renca/empty cells, which was not significantly enhanced by C5a treatment. This result suggests that, although $\mathrm{C} 5 \mathrm{aR}$ expression itself may have a marginal effect on cell survival in an adhesion-independent condition, it is unlikely that anoikis plays an important role in the metastasis of C5aR-expressing renal carcinoma cells.
C5a elicits cytoskeletal rearrangement and changes in cellular morphology in C5aR-expressing Renca cells. It is known that the chemoattractant $\mathrm{C} 5 \mathrm{a}$ causes actin rearrangement and stimulates the migration of leukocytes $(15,16)$. We previously showed that cancer cells can exploit this mechanism to acquire the ability of migration and invasion by activation of aberrantly expressed C5aR using bile duct carcinoma cells (9). To test the hypothesis that $\mathrm{C} 5 \mathrm{aR}$ expressed in renal cell carcinoma facilitates actin reorganization by $\mathrm{C} 5 \mathrm{a}$ stimulation as well, the effect of C5aR activation on actin rearrangement in Renca cells was analyzed by F-actin immunofluorescent staining. Without C5a stimulation, both Renca/empty and Renca/C5aR cells showed 


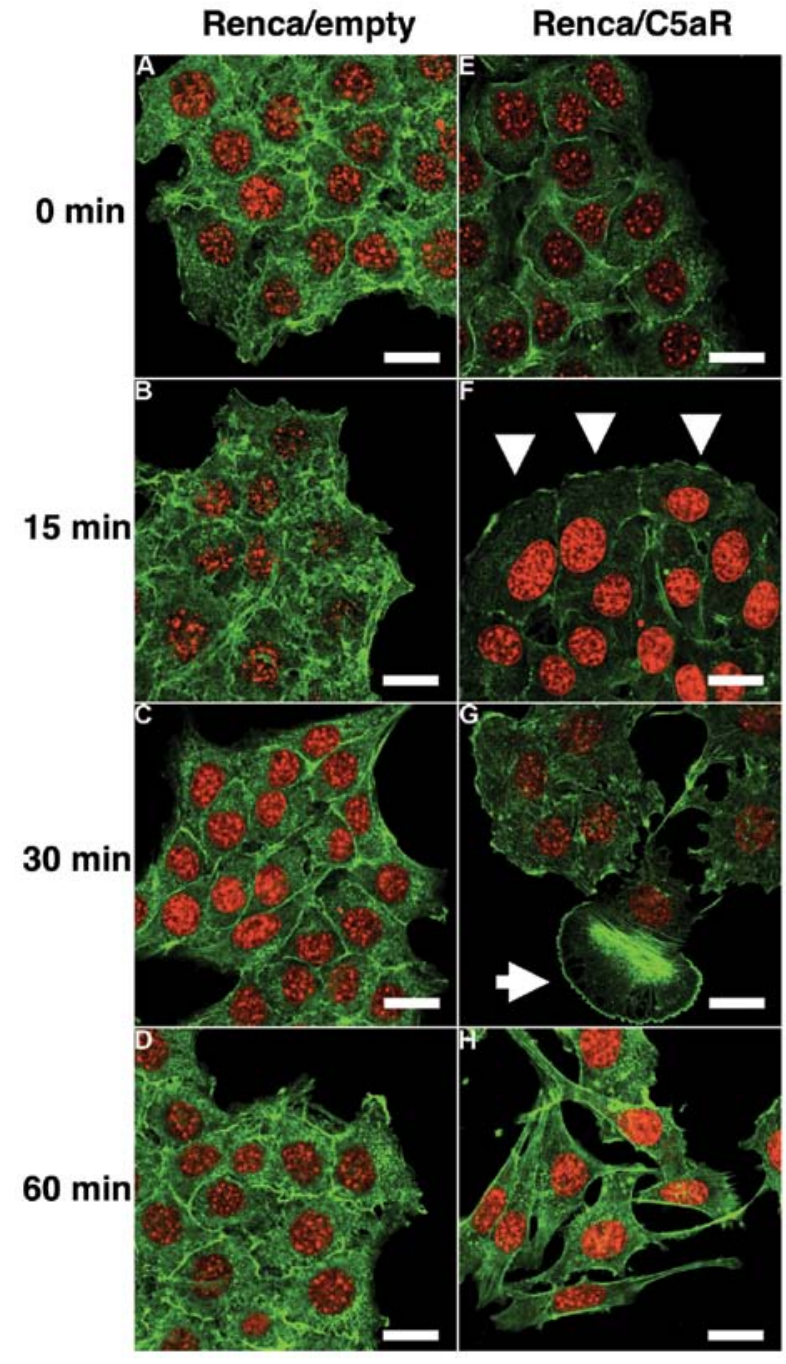

Figure 3. C5a elicits cytoskeletal rearrangement and changes in cellular morphology in the C5aR-expressing Renca cells. Renca/empty cells (A-D) and Renca/C5aR cells (E-G) were incubated with C5a (10 nM) and fixed at the indicated time-points. F-actin was visualized by immunofluorescent staining with Alexa 488-conjugated phalloidin (green), and nuclei with TO-PRO-3 (red). Scale bars, $20 \mu \mathrm{m}$. Arrowheads and the arrow indicate membrane ruffing and lamellipodia, respectively. C5aR, C5a receptor.

staining of cortical F-actin bundles at the borders of the cells (Fig. 3A and E). As early as 15 min after C5a stimulation, Renca/C5aR cells revealed membrane ruffling formation at the periphery of the cell clusters with reduced F-actin bundles at the cell-cell border (Fig. 3F). Thirty minutes after stimulation, the cell-cell contact became more loosened and some cells started to manifest lamellipodia formation (Fig. 3G). This was followed by marked change in cell shape such as stretched morphology and dissociation of cell clusters accompanied by stress fiber formation (Fig. 3H). In contrast, Renca/empty cells did not show any significant changes in both cytoskeleton and cellular morphology during observation despite C5a stimulation (Fig. 3A-D). These results suggest that C5a elicits cytoskeletal rearrangement and cellular morphological change in renal carcinoma cells via $\mathrm{C} 5 \mathrm{aR}$, leading to their dissociation and scattering.

C5a-C5aR axis enhances Renca cell invasion via the ERK and PI3K pathways. C5a is known to induce ERK $(5,17)$ and
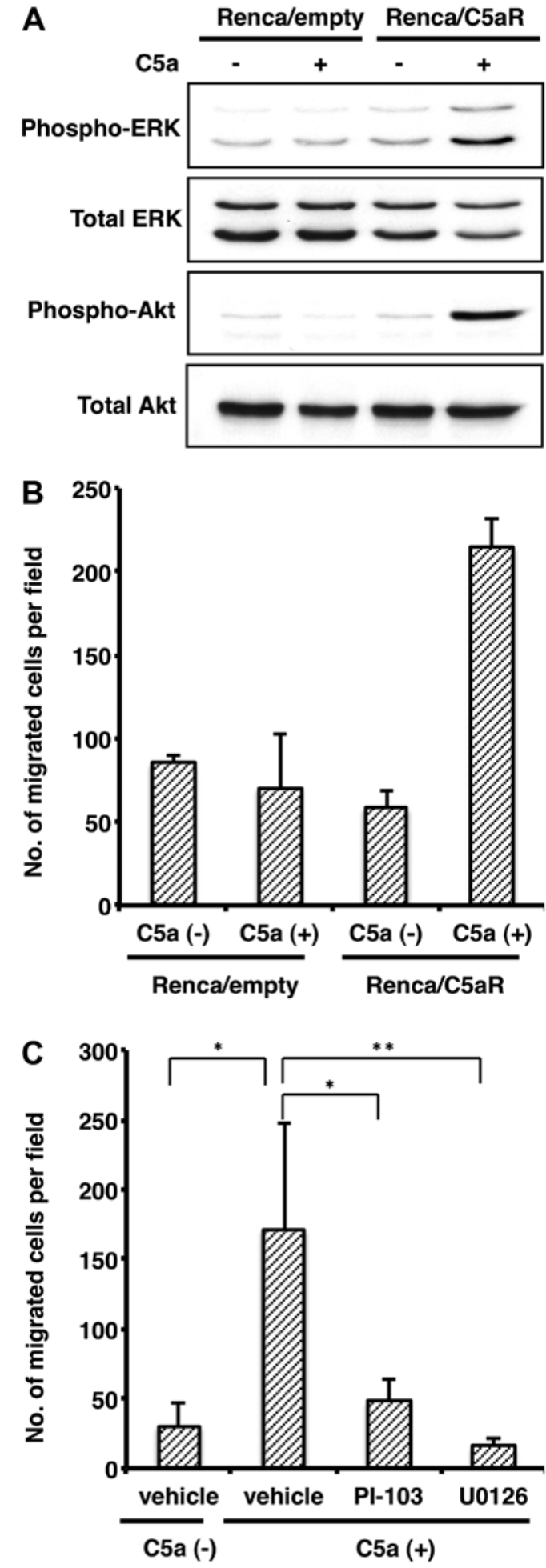

Figure 4. C5a-C5aR axis triggers ERK and PI3K-dependent cellular invasion. (A) Renca-derived cells were stimulated with or without $10 \mathrm{nM} \mathrm{C5a}$ for $15 \mathrm{~min}$ after serum starvation for $24 \mathrm{~h}$. Samples were harvested and subjected to immunoblotting using antibodies indicated. (B and C) Invasion assays were carried out using (B) Renca/empty and Renca/C5aR cells or (C) Renca/C5aR cells only. (C) Medium in both the upper and lower wells were mixed with the indicated kinase inhibitors or vehicle (DMSO). U0126 was used at $20 \mu \mathrm{M}$ and PI-103 was used at $0.5 \mu \mathrm{M}$. Data are presented as the mean \pm SD. ${ }^{*} \mathrm{P}<0.02$ and ${ }^{* *} \mathrm{P}<0.01$. C5aR, C5a receptor.

PI3K activation $(6,18)$ in inflammatory/immune cells in a C5aR-dependent manner, leading to manifestation of a variety 
of biological phenomena including leukocyte migration (19). Since ERK and PI3K are crucial mediators of extracellular stimuli-induced actin reorganization $(20,21)$ which was observed in Fig. 3, we investigated if C5a provokes ERK and PI3K activation in C5aR-expressing renal carcinoma cells. As shown in Fig. 4A, Renca/C5aR cells showed a robust increase in ERK and Akt, substrate of PI3K, phosphorylation by C5a stimuli, whereas such phosphorylation did not occur in the Renca-empty cells. This result indicates that the C5a-C5aR axis can activate ERK and PI3K kinase pathways in renal carcinoma cells. We previously reported that the C5a-C5aR axis enhances cancer cell invasion both in vitro and in vivo (9). To test if this is also the case in renal carcinoma cells, we performed an invasion assay using a Matrigel-coated Boyden chamber. Fig. 4B shows that C5a stimuli elicited invasion of the Renca/C5aR cells, but not the Renca-empty cells, in the Matrigel-coated boyden chamber, indicating that the C5a-C5aR axis can enhance invasion of renal cell carcinoma as well. In addition, treatment with either an MEK inhibitor U0126 or a PI3K inhibitor PI-103 significantly diminished Renca cell invasion promoted by the C5a-C5aR axis (Fig. 4C). This result indicates that the ERK and PI3K pathways are indispensable for C5a-triggered C5aR-expressing renal carcinoma cell invasion. All things considered, these results suggest that the C5a-C5aR axis elicits renal carcinoma cell metastasis by triggering actin reorganization and invasion induced by ERK and PI3K pathway activation.

\section{Discussion}

C5aR was originally identified in cells with a myeloid origin, and it has been shown by numerous studies that it mediates a wide variety of biological phenomena in myeloid cells induced by $\mathrm{C} 5 \mathrm{a}$ such as leukocyte migration upon inflammation. However, Cao et al (5) reported an intriguing finding that the $\mathrm{C} 5 \mathrm{aR}$ is also expressed in epithelial cells, which suggested the involvement of $\mathrm{C} 5 \mathrm{aR}$ in other biological processes in nonmyeloid cells. Recently, we showed that C5aR is aberrantly expressed in various types of human cancers (9), which was the first study regarding the biological significance of C5aR expression in cancer cells. In that study, we also showed that $\sim 60 \%$ of RCC specimens expressed C5aR. However, the sample number of RCC specimens in that study was limited $(n=11)$ and not sufficient to analyze the relationship between C5aR expression status and clinical parameters. In this study, we investigated $127 \mathrm{RCC}$ samples using immunohistochemistry and found a similar frequency of C5aR expression $(61.4 \%$; 78/127) as in the previous study. It is of note that around half of adjacent normal kidney tubular epithelial cells already express C5aR (9), which was confirmed in this expanded analysis (data not shown). At the moment, the biological significance of $\mathrm{C} 5 \mathrm{aR}$ expression in normal renal tubular epithelial cells is unknown. The fact that around $40 \%$ of RCC samples (49/127) did not express $\mathrm{C} 5 \mathrm{aR}$, implies that $\mathrm{C} 5 \mathrm{aR}$ could be a sublineage marker of renal tubular epithelial cells and the expression status of C5aR in renal cell carcinoma may be stochastic and reflect its sublineage rather than a consequence of malignant transformation. Angelotti et al (22) reported that there are 2 subpopulations of renal progenitors with the potential to regenerate tubular epithelial cells. It would be intriguing if
C5a-positive renal tubular epithelial cells represent either of those populations.

In the study cohort we examined by immunohistochemistry, C5aR-positive RCCs manifested both a locally invasive and metastatic phenotype with a higher incidence of microvascular invasion. In addition, we demonstrated that $\mathrm{C} 5 \mathrm{a}-\mathrm{C} 5 \mathrm{aR}$ axis activation elicited invasion using an in vitro invasion assay with a renal carcinoma cell line. Hence C5aR appears to facilitate local invasion to adjacent tissues and microvascular infiltration thereby promoting distant metastasis of renal carcinoma cells. Generally, metastasis requires a number of steps including dissociation of cancer cells from primary sites, invasion through basement membrane and into blood/lymphatic vessels, survival when floating in blood/lymphatic stream, and implantation and proliferation in distant target organs (23). From both clinical and experimental results shown in the present study, it is plausible that $\mathrm{C} 5 \mathrm{aR}$ expression contributes at least to the invasion steps in this metastasis model.

We performed renal subcapsular injection of Renca-derived cells in $\mathrm{BALB} / \mathrm{c}$ mice to examine if $\mathrm{C} 5 \mathrm{aR}$ expression promotes spontaneous renal carcinoma cell metastasis in a syngeneic orthotopic murine model. Although we were able to observe lung metastasis using this model as reported in the literature (11), we could not find any difference in either the size or the number of metastatic foci in the lung between Renca/empty and Renca/C5aR cells regardless of recombinant C5a treatment. Our previous study showed an increased invasion of subcutaneously injected C5aR-expressing HuCCT1 cells in nude mice compared to HuCCT1 cells harboring empty plasmid (9). In the latter assay, the 2-day period was sufficient to observe a significant increase in local invasion. However, in the case of the syngeneic orthotopic murine model used in the present study, it required up to 3 weeks to observe spontaneous lung metastasis (11), which may be sufficiently long to offset the effect of the recombinant C5a stimulation to Renca/C5aR cells before injection. In order to appropriately assess the effect of C5a-C5aR axis activation on spontaneous metastasis in this murine model, improved experimental methods may be required for sustained activation of $\mathrm{C} 5 \mathrm{aR}$.

Acquisition of anoikis resistance by cancer cells is also an important step for establishing cancer metastasis because cancer cells have to survive in the floating condition during their travel through the circulatory and lymphatic systems until implantation to target organs (14). These facts led us to analyze further whether C5a-C5aR axis activation contributes to this cellular phenomenon. However, C5a stimulation did not have a significant impact on anoikis resistance in the renal carcinoma cells regardless of $\mathrm{C} 5 \mathrm{aR}$ expression. Therefore we concluded that the $\mathrm{C} 5 \mathrm{a}-\mathrm{C} 5 \mathrm{aR}$ axis does not play a significant role in acquiring anoikis-resistance in $\mathrm{mRCC}$.

We showed that C5a induced dynamic reorganization of actin cytoskeleton in the C5aR-expressing renal carcinoma cells, namely, lamellipodia and stress fiber formation, resulting in dissociation of cell clusters and scattering. It is well known that these processes are mediated by activation of Rho family small G proteins such as Rac1 and RhoA (24). Li et al (25) previously reported that $\mathrm{C} 5 \mathrm{a}$ induced activation of Rho family small $\mathrm{G}$ proteins in neutrophils, leading to actin reorganization of the cell, suggesting that the C5a-C5aR axis is one of the upstream switches of Rho family protein activation. It would 
be of interest to analyze if Rho family proteins are activated when actin rearrangement is induced in renal carcinoma cells expressing C5aR by C5a stimulation.

In addition to inducing actin rearrangement, C5a elicited invasion of C5aR-expressing renal carcinoma cells in a Matrigel-coated Boyden chamber. Therefore, it is plausible that C5a-C5aR may be involved in the metastasis of renal cell carcinoma by prompting dissociation of cancer cells from primary sites and invasion through the basement membrane. Furthermore, C5a stimulation activated the ERK and PI3K pathways and inhibition of these kinase pathways by specific inhibitors negated the C5aR-expressing renal carcinoma cell invasion. These pathways are known to be activated by $\mathrm{C} 5 \mathrm{a}$ stimulation in cells with myeloid origin to regulate numerous biological phenomena (19). In the present study, we showed that such activation does occur in renal carcinoma cells. This is the first study to show that the C5a-C5aR axis does trigger activation of these kinase pathways and invasion in cancer cells. Campbell et al (26) reported that phosphorylated ERK is an independent prognostic biomarker that significantly predicts the onset of metastasis in clinically confined RCC, and Horiguchi et al (27) showed that phosphorylated Akt is significantly associated with RCC metastasis. These studies are consistent with our finding that $\mathrm{C} 5 \mathrm{aR}$, which can trigger ERK and PI3K activation by C5a stimulation, is expressed in a vast majority of clinical mRCC specimens.

The present study provides the proof-of-concept that the C5a-C5aR axis can be a novel target for preventing renal cell carcinoma progression as well as further support of the current therapeutic concept to target the ERK and PI3K/mTOR pathways in mRCC (28). Clinical application of this concept may contribute to develop novel therapeutic strategies for advanced $\mathrm{RCC}$ in the future.

\section{Acknowledgements}

We thank S. Nakata, M. Matsumoto and T. Kubo for technical assistance. We are also grateful to K. Yoshinobu, The Gene Technology Center in Kumamoto University for her important contributions to the immunofluorescence experiments.

\section{References}

1. Zisman A, Pantuck AJ, Wieder J, et al: Risk group assessment and clinical outcome algorithm to predict the natural history of patients with surgically resected renal cell carcinoma. J Clin Oncol 20: 4559-4566, 2002.

2. Wada Y, Takahashi W, Kawano Y and Eto M: Current status of pharmacotherapy against metastatic renal cell carcinoma in Japan. Int J Urol 19: 284-295, 2012.

3. Müller A, Homey B, Soto H, et al: Involvement of chemokine receptors in breast cancer metastasis. Nature 410: 50-56, 2001.

4. Guo RF and Ward PA: Role of C5a in inflammatory responses. Annu Rev Immunol 23: 821-852, 2005.

5. Cao Q, McIsaac SM and Stadnyk AW: Human colonic epithelial cells detect and respond to C5a via apically expressed C5aR through the ERK pathway. Am J Physiol Cell Physiol 302: C1731-C1740, 2012.

6. Kwan WH, van der Touw W, Paz-Artal E, Li MO and Heeger PS Signaling through $\mathrm{C} 5$ a receptor and $\mathrm{C} 3$ a receptor diminishes function of murine natural regulatory T cells. J Exp Med 210: 257-268, 2013.
7. Chiou WF, Tsai HR, Yang LM and Tsai WJ: C5a differentially stimulates the ERK1/2 and p38 MAPK phosphorylation through independent signaling pathways to induced chemotactic migration in RAW264.7 macrophages. Int Immunopharmacol 4: 1329-1341, 2004.

8. Tsai HR, Yang LM, Tsai WJ and Chiou WF: Andrographolide acts through inhibition of ERK1/2 and Akt phosphorylation to suppress chemotactic migration. Eur J Pharmacol 498: 45-52, 2004.

9. Nitta H, Wada Y, Kawano Y, et al: Enhancement of human cancer cell motility and invasiveness by anaphylatoxin C5a via aberrantly expressed C5a receptor (CD88). Clin Cancer Res 19: 2004-2013, 2013.

10. Kawano Y, Diez S, Uysal-Onganer P, Darrington RS, Waxman J and Kypta RM: Secreted frizzled-related protein-1 is a negative regulator of androgen receptor activity in prostate cancer. Br J Cancer 100: 1165-1174, 2009.

11. Shvarts O,Janzen N,Lam JS, et al: RENCA/carbonic anhydrase-IX: a murine model of a carbonic anhydrase- IX-expressing renal cell carcinoma. Urology 68: 1132-1138, 2006.

12. Berezovskaya O, Schimmer AD, Glinskii AB, et al: Increased expression of apoptosis inhibitor protein XIAP contributes to anoikis resistance of circulating human prostate cancer metastasis precursor cells. Cancer Res 65: 2378-2386, 2005.

13. Eto M, Kamiryo Y, Takeuchi A, et al: Posttransplant administration of cyclophosphamide and donor lymphocyte infusion induces potent antitumor immunity to solid tumor. Clin Cancer Res 14: 2833-2840, 2008.

14. Frisch SM and Screaton RA: Anoikis mechanisms. Curr Opin Cell Biol 13: 555-562, 2001.

15. Banks P, Barker MD and Burton DR: Recruitment of actin to the cytoskeletons of human monocyte-like cells activated by complement fragment $\mathrm{C} 5 \mathrm{a}$. Is protein kinase $\mathrm{C}$ involved? Biochem J 252: 765-769, 1988.

16. Monk PN and Banks P: Evidence for the involvement of multiple signalling pathways in $\mathrm{C} 5 \mathrm{a}$-induced actin polymerization and nucleation in human monocyte-like cells. J Mol Endocrinol 6: 241-247, 1991.

17. Li K, Fazekasova H, Wang N, et al: Functional modulation of human monocytes derived DCs by anaphylatoxins C3a and C5a. Immunobiology 217: 65-73, 2012.

18. Bosmann M, Patel VR, Russkamp NF, et al: MyD88-dependent production of IL-17F is modulated by the anaphylatoxin C5a via the Akt signaling pathway. FASEB J 25: 4222-4232, 2011.

19. Don MJ, Liao JF, Lin LY and Chiou WF: Cryptotanshinone inhibits chemotactic migration in macrophages through negative regulation of the PI3K signaling pathway. Br J Pharmacol 151: 638-646, 2007.

20. Mendoza MC, Er EE, Zhang W, et al: ERK-MAPK drives lamellipodia protrusion by activating the WAVE2 regulatory complex. Mol Cell 41: 661-671, 2011.

21. Posern G, Saffrich R, Ansorge W and Feller SM: Rapid lamellipodia formation in nerve growth factor-stimulated PC12 cells is dependent on Rac and PI3K activity. J Cell Physiol 183: 416-424, 2000.

22. Angelotti ML, Ronconi E, Ballerini L, et al: Characterization of renal progenitors committed toward tubular lineage and their regenerative potential in renal tubular injury. Stem Cells 30: 1714-1725, 2012.

23. Hanahan D and Weinberg RA: Hallmarks of cancer: the next generation. Cell 144: 646-674, 2011.

24. Hall A: Rho GTPases and the actin cytoskeleton. Science 279: 509-514, 1998.

25. Li Z, Hannigan M, Mo Z, et al: Directional sensing requires $\mathrm{G}$ beta gamma-mediated PAK1 and PIX alpha-dependent activation of Cdc42. Cell 114: 215-227, 2003.

26. Campbell L, Nuttall R, Griffiths D and Gumbleton M: Activated extracellular signal-regulated kinase is an independent prognostic factor in clinically confined renal cell carcinoma. Cancer 115: 3457-3467, 2009.

27. Horiguchi A, Oya M, Uchida A, Marumo K and Murai M: Elevated Akt activation and its impact on clinicopathological features of renal cell carcinoma. J Urol 169: 710-713, 2003.

28. Figlin RA, Kaufmann I and Brechbiel J: Targeting PI3K and mTORC2 in metastatic renal cell carcinoma: new strategies for overcoming resistance to VEGFR and mTORC1 inhibitors. Int J Cancer 133: 788-796, 2013. 